\title{
The efficacy and complications of computed tomography guided microwave ablation in lung cancer
}

\author{
Bin Chen ${ }^{1 \#}$, Wei Li ${ }^{1 \#}$, Yu Liu ${ }^{1,2 \#}$, Shengxiang Ren ${ }^{1}$, Lei Wang ${ }^{1}$, Hao Wang ${ }^{1,2}$, Sha Zhao ${ }^{1}$, Lingyun Ye ${ }^{1}$, \\ Yayi $\mathrm{He}^{1}$, Caicun Zhou ${ }^{1}$ \\ ${ }^{1}$ Department of Medical Oncology, Shanghai Pulmonary Hospital, Tongji University Medical School Cancer Institute, Tongji University School of \\ Medicine, Shanghai, China; ${ }^{2}$ Tongji University, Shanghai, China \\ Contributions: (I) Conception and design: B Chen, W Li, Y Liu; (II) Administrative support: Y He, C Zhou; (III) Provision of study materials or \\ patients: S Ren, L Wang; (IV) Collection and assembly of data: H Wang, S Zhao, L Ye; (V) Data analysis and interpretation: B Chen, W Li, Y Liu; (VI) \\ Manuscript writing: All authors; (VII) Final approval of manuscript: All authors. \\ \#These authors contributed equally to this work. \\ Correspondence to: Yayi He; Caicun Zhou. Department of Medical Oncology, Shanghai Pulmonary Hospital, Tongji University Medical School Cancer \\ Institute, Tongji University School of Medicine, No 507 Zhengmin Road, Shanghai 200433, China. \\ Email: 2250601@qq.com; caicunzhoudr@126.com.
}

\begin{abstract}
Background: This study aimed to explore the effect and safety of computed tomography (CT) guided microwave ablation for lung cancer.

Methods: We retrospectively enrolled CT guided microwave ablation of lung cancer patients in Shanghai Pulmonary Hospital from March 2016 to September 2019. All patients were pathologically confirmed by fine needle biopsy before microwave ablation. We reviewed CT imaging of 45 patients with lung cancer before and after microwave ablation and recorded the complications. Date were analyzed by paired-samples T-test and one-way analysis of variance.

Results: In 45 lung cancer patients received CT guided microwave ablation, the tumor diameter changed from $2.35 \pm 0.22 \mathrm{~cm}$ before surgery to $2.26 \pm 0.19 \mathrm{~cm}$ after surgery, average reduction by $0.09 \mathrm{~cm}(\mathrm{P}=0.518)$. With microwave ablation treatment, the disease control rate reached $82.93 \%$, and the incidence of pneumothorax was $13.3 \%$, with no other related adverse. The successful rate of operation was $100 \%$, and there was no severe or death related adverse.
\end{abstract}

Conclusions: CT-guided microwave ablation is an effective method in the treatment of lung cancer, with a low incidence of complications. It is a safe and effective method for the treatment of lung cancer.

Keywords: Microwave ablation; lung cancer; safety; effect

Submitted Jan 18, 2020. Accepted for publication Apr 09, 2020.

doi: $10.21037 /$ apm-20-255

View this article at: http://dx.doi.org/10.21037/apm-20-255

\section{Introduction}

According to GLOBOCAN, there are 2.1 million new lung cancer cases worldwide, accounting for $11.6 \%$ of all new cancer cases. The incidence of lung cancer ranks first among all cancers in most countries (1). Nowadays, surgical treatment is still the primary method for early stage lung cancer (2), but many patients are too late to have chance of surgery $(3,4)$. Chemotherapy plays an important role in treatment, but the side effects were high (5). Only some specific populations could get benefits from targeted therapy or immunotherapy $(6,7)$. With the improvement of modern medical technology, the thermal ablation has offered another treatment choice. Microwave ablation is a minimally invasive therapeutic approach, which is more and more widely used in lung cancer, primary liver cancer and other solid tumor and has good therapeutic outcomes (8-11). It could be performed in some medical institutions or even by outpatient surgery, with almost no absolute 
Table 1 Clinical characteristics of patients undergoing microwave ablation to treat pulmonary malignancies

\begin{tabular}{|c|c|}
\hline Characteristic & Total \\
\hline \multicolumn{2}{|l|}{ Age, $\mathrm{n}(\%)$} \\
\hline$<70$ years & $25(55.6)$ \\
\hline$\geq 70$ years & $20(44.4)$ \\
\hline \multicolumn{2}{|l|}{ Gender, n (\%) } \\
\hline Male & $26(57.8)$ \\
\hline Female & $19(42.2)$ \\
\hline \multicolumn{2}{|l|}{ Smoking status, n (\%) } \\
\hline Non-smoker & $26(57.8)$ \\
\hline Smoker & $19(42.2)$ \\
\hline \multicolumn{2}{|l|}{ Pathology, n (\%) } \\
\hline SCC & $3(6.7)$ \\
\hline Adenocarcinoma & $25(55.6)$ \\
\hline Squamous carcinoma & $10(22.2)$ \\
\hline NSCLC-NOS & $7(1.6)$ \\
\hline \multicolumn{2}{|l|}{ Lung cancer staging, $\mathrm{n}(\%)$} \\
\hline I & $13(28.9)$ \\
\hline II & $2(4.4)$ \\
\hline III & $5(11.1)$ \\
\hline IV & $16(35.6)$ \\
\hline Unknown stage & $9(20.0)$ \\
\hline \multicolumn{2}{|l|}{ Efficacy, n (\%) } \\
\hline $\mathrm{CR}$ & $4(9.76)$ \\
\hline PR & $5(12.20)$ \\
\hline SD & $25(60.98)$ \\
\hline PD & $7(17.07)$ \\
\hline \multicolumn{2}{|l|}{ Complications, n (\%) } \\
\hline None & $39(86.7)$ \\
\hline Pneumothorax & $6(13.3)$ \\
\hline
\end{tabular}

SCC, small cell lung cancer; NSCLC-NOS, non-small cell lung cancer- not otherwise specified; CR, complete response; PR, partial response; $\mathrm{SD}$, stable disease; $\mathrm{PD}$, progressive disease.

contraindications except for uncorrectable coagulation dysfunction, thrombocytopenia or the tumor adjacent to an important anatomical structure (12). Hence, microwave ablation was worth exploring its application further. In this paper, we analyzed the effect and safety of CT guided microwave ablation in 45 lung cancer patients.

\section{Methods \\ Patients}

We retrospectively enrolled 45 CT guided microwave ablation of lung cancer patients in Shanghai Pulmonary Hospital from March 2016 to September 2019. Approval of this study was obtained from Shanghai Pulmonary Hospital Ethical Inspection Committee (ethical number $18 \mathrm{Q} 016 \mathrm{NJ})$. This study was conducted in accordance with the Declaration of Helsinki (as revised in 2013). Written informed consent was obtained from the patient for publication of this manuscript and any accompanying images. All patients' malignancies were considered medically inoperable or patients refused surgery. Patient characteristics were shown in Table 1 . The patients included 26 males $(57.8 \%)$ and 19 females (42.2\%), with the median age of 68 . Almost all pathological types and stages were involved. There were $25(55.6 \%)$ adenocarcinoma, 10 (22.2\%) squamous carcinoma and $3(6.7 \%)$ small cell lung cancer and 7 (1.6\%) non-small cell lung cancer-not otherwise specified (NSCLC-NOS). And as for pathological stage, there were 13 (28.9\%) cases of stage I, 2 (4.4\%) cases of stage II, $5(11.1 \%)$ cases of stage III, $16(35.6 \%)$ cases of stage IV and $9(20.0 \%)$ unknown stage.

\section{Procedure}

Siemens 64 slice spiral CT machines were used. The microwave ablation system was consisted of generator and microwave antenna. The microwave generator (Spherical Water-cooled Microwave Ablation System; Vision Medical USA Inc.) that was used was capable of producing up to $40-60 \mathrm{~W}$ of power. We used a 17-gauge monopolar cooled microwave needle. The length of the range was from 0 to $15 \mathrm{~cm}$ (depending on the depth of the lesion treatment) and the non-insulated portion of the needle was between 1 to $3 \mathrm{~cm}$. Preoperative interview and preoperative examination were conducted. Before the operation, we asked patients to breathe smoothly. Then we scanned tumor position by CT and decided the needle direction and angle. Under local anesthesia, the puncture needle was slowly inserted into the center of the tumor. Finally, it was connected to an emitter. The specific time of microwave ablation was determined according to the lesion sizes of the patients. For patients with larger lesions, multiple sites were applied for superposition ablation. If the diameter of the tumor was less 


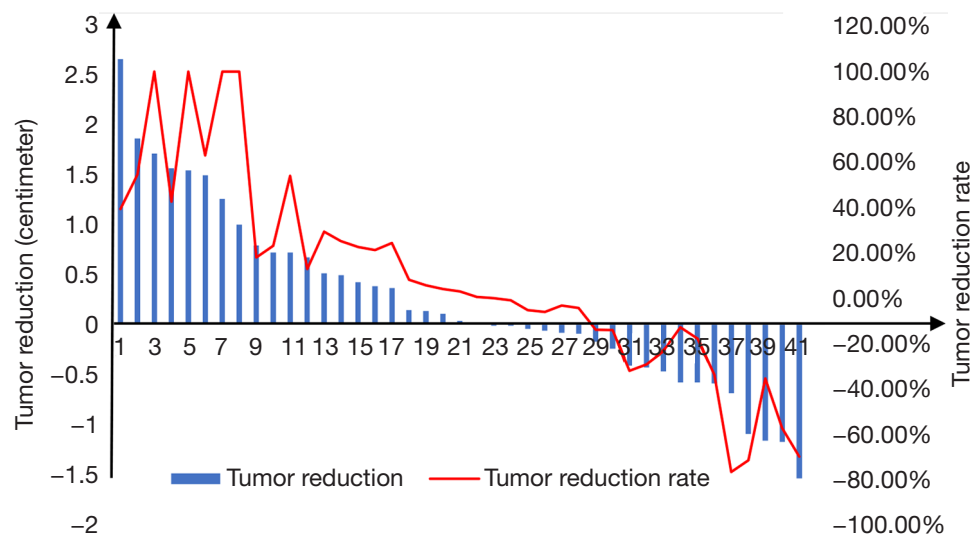

Figure 1 Tumor reduction and reduction rate after microwave ablation therapy.

than $3 \mathrm{~cm}$, the insertion point could be inserted into the center of the tumor nodules, and then microwave ablation could be performed. And if larger than $3 \mathrm{~cm}$ in diameter, the shape of tumor nodules was generally irregular, in this case the position of the tip could be adjusted gradually until the whole tumor nodules were removed by microwave ablation. After the ablation, the needles way was ablated to prevent needle implantation. After the completion of treatment, CT scan was performed again to observe the pneumothorax, bleeding and other complications. Then the patient was taken to return to the ward and laid for 2 hours. Chest X-ray was reexamined 1 week after surgery and CT scan was performed one month later.

\section{Data analysis}

Statistical analysis was processed by SPSS 25.0. Numerical data were expressed in terms of case number and constituent ratio. The size of tumor before and after microwave ablation was statistically analyzed by paired-samples T-test. And the possible influencing factors of efficacy and complications were analyzed by one-way analysis of variance. $\mathrm{P}<0.05$ indicated that the difference was statistically significant.

\section{Results}

All the 45 patients with lung cancer were successfully completed microwave ablation. The tumor diameter changed from $2.35 \pm 0.22 \mathrm{~cm}$ before surgery to $2.26 \pm$ $0.19 \mathrm{~cm} 1$ month after surgery, average reduction by $0.09 \mathrm{~cm}$ $(\mathrm{P}=0.518)$. The tumor reduction and the rate showed in Figure 1 (four patients were excluded for efficacy analysis due to loss of follow-up, and only their complication rates were analyzed). With microwave ablation treatment, the disease control rate was $82.93 \%$, which was calculated by complete response (CR) plus partial response (PR) plus stable disease (SD). Four patients were re-examined 1 month after microwave ablation and found cavitation in the original tumor site (Figure 2). There was no intraoperative death or severe adverse cases. Postoperative complications included pneumothorax $(n=9,13.3 \%)$, no pleural effusion, fever, hemorrhage, hemoptysis and pneumonic phrenic nerve injury. After symptomatic treatment, the related complications were improved and the patients could tolerate them. Additionally, we could observe that the lesion showed decreased CT density after ablation in the CT image.

However, we found that there was no significant difference in the efficacy among the different pathological stages $(\mathrm{P}=0.613)$, different pathological types $(\mathrm{P}=0.862)$ and different age groups $(\mathrm{P}=0.375)$. And there was also no significant difference in the complication rates among the different pathological stages $(\mathrm{P}=0.182)$, different pathological types $(\mathrm{P}=0.389)$ and different age groups $(\mathrm{P}=0.249)$. In addition, there was no correlation between the efficacy and clinical factors such as age, gender, smoking history, and tumor size before microwave ablation in this study.

\section{Discussion}

Studies showed that resection of lung tumors that met the surgical indications could achieve a satisfactory effect of extending the survival time, but only a small part of lung cancer patients can get the surgery (13). Because some patients with lung cancer could not be treated by 

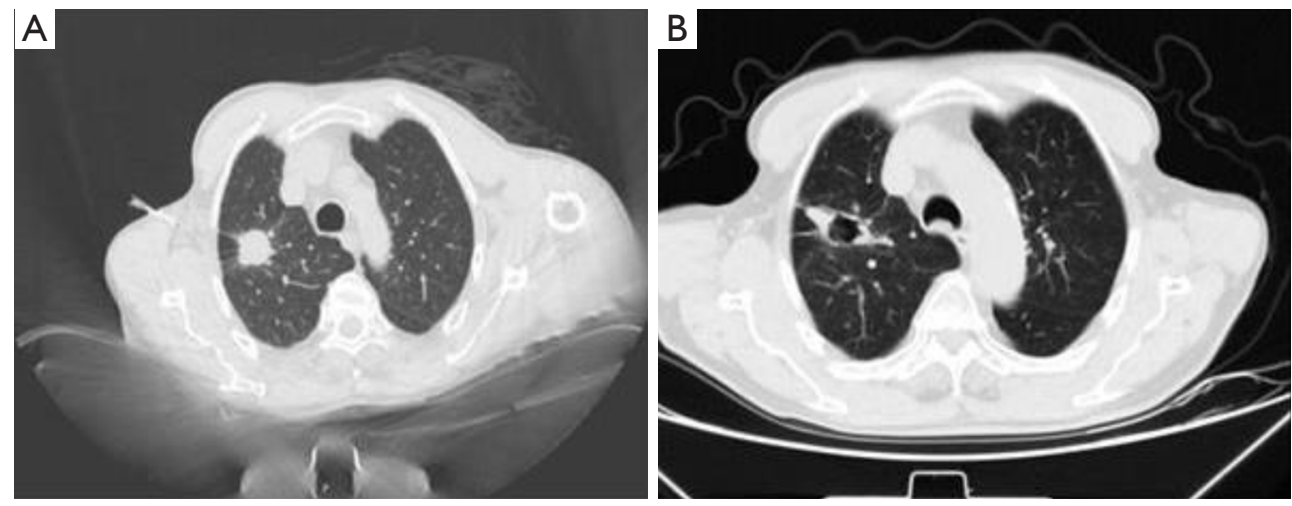

Figure 2 Cavitation of microwave ablation treatment. (A) The CT image before microwave ablation; (B) the CT image after microwave ablation.

radiotherapy and surgery, microwave ablation offered an opportunity to cure it. Dupuy et al. applied radiofrequency ablation to the treatment of lung cancer in 2000, opened a new era of minimally invasive treatment for cancer (14). Several studies indicated that microwave ablation might be a more effective method to treat solid tumors $(15,16)$ and had a similar complication incidence with radiofrequency ablation (17). Microwave thermal ablation was to take advantage of the thermal effect of microwaves and the fact that tumor cells were not heat resistant. It emitted microwaves $(2,450$ or $915 \mathrm{mHz})$ through the puncture needle in the tumor, so that water molecules in the tissue could absorb the microwave energy and generate heat by high-speed motion, oscillation and friction. Because of the rapid rate of microwave temperature rise, the local temperature in the tumor lesion could exceed $100^{\circ} \mathrm{C}$ in a very short time, resulting in coagulation necrosis of the protein of the tumor cells, so as to inactivate the tumor tissue and achieve the purpose of treatment. Inflammation could further lead to tumor necrosis after treatment (18). Ablation of tumor cell fragments may also lead to systemic tumor control by initiating or enhancing tumor-specific adaptive immunity (19). In addition, the low blood flow of lung tumor tissues made microwave ablation feasible and effective in the treatment of lung tumors (20).

More and more evidence showed that microwave ablation was better than radiofrequency ablation. In 2017, Nour-Eldin et al. retrospectively compared the treatment results of microwave ablation, radiofrequency ablation and laser ablation for lung metastatic tumor, and concluded that microwave ablation was superior to the others in terms of survival rate and local control rate (21). Radiofrequency ablation was susceptible to the cooling effect (radiator effect) of air or blood movement, but microwave heat transfer was less affected. This allowed microwave ablation to produce a larger ablation area, reducing surgical time, and improving outcomes for cystic lesions or lesions near vascular structures $(22,23)$. Besides, radiofrequency electrodes were prone to carbonization and adhesion to tumor tissue, then the electrode cannot be retracted. And the mechanism of microwave ablation avoided it.

In our study, the disease control rate of microwave ablation was $82.93 \%$. Cavitation occurred in four patients after microwave ablation. It was reported that cavitation in treated tumors was correlated with reduced cancer-specific mortality (17). Wolf et al. reported that 82 metastatic lung cancers were treated by CT-guided percutaneous microwave ablation in 50 patients, $74 \%$ of the microwave ablation sites were completely ablated, $78 \%$ of the patients had no tumor recurrence, the local control rate was $67 \%$ at 1 year (17), which was roughly in line with our data. Yashiro et al. treated 210 lung tumors in 71 patients with CT-guided percutaneous microwave ablation, and the local tumor progression-free rate at 1,2 and 3 years was $80.4 \%, 69.0 \%$ and $67.7 \%$ respectively (24).

In this study, no microwave ablation-related death occurred and the success rate of microwave ablation was $100 \%$. Postoperative complications were only pneumothorax in $13.3 \%$ patients. The incidence of complications in this group was low, which might be correlated with our selective ablation strategies, especially the selection of puncture protocol. Doctor's skilled puncture technique might also play an important role in this success. Pneumothorax was one of the most common complications of microwave ablation, literature reported the rate was 10-67\% (25). However, merely $3.8-15.7 \%$ of the pneumothorax required intercostal catheters (23). There were also reports of late pneumothorax occurring on the $2-5$ day after radiofrequency ablation (26), 
so it was necessary to perform X-ray and confirm the presence of pneumothorax. Pleural effusion was also a common complication, which was thought to be the body's sympathetic response to heat damage. And Wolf etal. reported a $0.7 \%$ incidence rate of effusion, with no one requiring drainage (17). The incidence of pulmonary infection caused by ablation was 1-6\% (27). However, we could not ignore the fact that most of the patients with lung cancer were elderly, who could not tolerate surgical treatment and were often accompanied by basic lung diseases. Pulmonary infection and inflammation might lead to a sharp decline in lung function and even death of the patients, which need more attention from doctors. Bleeding was not common because of hemostatic effect of thermal ablation. Microwave ablation may also cause damage to peripheral nerve tissue, such as brachial plexus injury, phrenic nerve injury and phrenic hernia, but they were rare (27). Bronchopleural fistula, acute respiratory distress syndrome, rib fracture, pulmonary embolism and air embolism also have been reported as rare cases. Most of the above complications could be improved after symptomatic treatment, and patients could be well tolerated. There were several limitations of our study. First, it was a retrospective research. And the number of clinical cases included in our study was a little small. Additionally, the follow-up time was not enough to observe the endpoint.

\section{Conclusions}

CT guided percutaneous microwave ablation was demonstrated to be an effective means of local physical therapy for lung cancer in our study, and its advantages of less trauma and low complication rate could be applied in more patients who could not receive surgical treatment. In summary, microwave ablation is a recommended promising technique. We will enroll more patients in our prospective trial and conduct longer follow-up to obtain survival data.

\section{Acknowledgments}

Funding: This study was supported in part by a grant from National Natural Science Foundation of China (81802255), Shanghai Pujiang Program (17PJD036) and a grant from Shanghai Municipal Commission of Health and Family Planning Program (20174Y0131), National Key Research \& Development Project (2016YFC0902300), Major Disease Clinical Skills Enhancement Program of Three Year Action Plan for Promoting Clinical Skills and Clinical Innovation in Municipal Hospitals, Shanghai Shen
Kang Hospital Development Center Clinical Research Plan of SHDC (16CR1001A), "Dream Tutor" Outstanding Young Talents Program (fkyq1901), Key Disciplines of Shanghai Pulmonary Hospital (2017ZZ02012), grant of Shanghai Science and Technology Commission (16JC1405900).

\section{Footnote}

Conflicts of Interest: All authors have completed the ICMJE uniform disclosure form (available at http://dx.doi. org/10.21037/apm-20-255). The authors have no conflicts of interest to declare.

Ethical Statement: The authors are accountable for all aspects of the work in ensuring that questions related to the accuracy or integrity of any part of the work are appropriately investigated and resolved. Approval of this study was obtained from Shanghai Pulmonary Hospital Ethical Inspection Committee (ethical number 18Q016NJ). This study was conducted in accordance with the Declaration of Helsinki (as revised in 2013). Written informed consent was obtained from the patient for publication of this manuscript and any accompanying images.

Open Access Statement: This is an Open Access article distributed in accordance with the Creative Commons Attribution-NonCommercial-NoDerivs 4.0 International License (CC BY-NC-ND 4.0), which permits the noncommercial replication and distribution of the article with the strict proviso that no changes or edits are made and the original work is properly cited (including links to both the formal publication through the relevant DOI and the license). See: https://creativecommons.org/licenses/by-nc-nd/4.0/.

\section{References}

1. Bray F, Ferlay J, Soerjomataram I, et al. Global cancer statistics 2018: GLOBOCAN estimates of incidence and mortality worldwide for 36 cancers in 185 countries. CA Cancer J Clin 2018;68:394-424.

2. Scott WJ, Howington J, Feigenberg S, et al. Treatment of non-small cell lung cancer stage I and stage II: ACCP evidence-based clinical practice guidelines (2nd edition). Chest 2007;132:234s-42s.

3. He Y, Sun W, Wang Y, et al. Comparison of erlotinib and pemetrexed as second-/third-line treatment for lung adenocarcinoma patients with asymptomatic brain 
metastases. Onco Targets Ther 2016;9:2409-14.

4. He Y, Wang Y, Boyle T, et al. Hepatic Metastases is Associated with Poor Efficacy of Erlotinib as 2nd/3rd Line Therapy in Patients with Lung Adenocarcinoma. Med Sci Monit 2016;22:276-83.

5. Zhang QY, Wang FX, Jia KK, et al. Natural Product Interventions for Chemotherapy and RadiotherapyInduced Side Effects. Front Pharmacol 2018;9:1253.

6. He Y, Liu S, Mattei J, et al. The combination of anti-KIR monoclonal antibodies with anti-PD-1/PD-L1 monoclonal antibodies could be a critical breakthrough in overcoming tumor immune escape in NSCLC. Drug Des Devel Ther 2018;12:981-6.

7. He Y, Yu H, Rozeboom L, et al. LAG-3 Protein Expression in Non-Small Cell Lung Cancer and Its Relationship with PD-1/PD-L1 and Tumor-Infiltrating Lymphocytes. J Thorac Oncol 2017;12:814-23.

8. Goldberg SN, Gazelle GS, Mueller PR. Thermal ablation therapy for focal malignancy: a unified approach to underlying principles, techniques, and diagnostic imaging guidance. AJR Am J Roentgenol 2000;174:323-31.

9. Seki T, Wakabayashi M, Nakagawa T, et al. Percutaneous microwave coagulation therapy for solitary metastatic liver tumors from colorectal cancer: a pilot clinical study. Am J Gastroenterol 1999;94:322-7.

10. Simon CJ, Dupuy DE, Mayo-Smith WW. Microwave ablation: principles and applications. Radiographics 2005;25 Suppl 1:S69-83.

11. Filippiadis DK, Gkizas C, Chrysofos M, et al. Percutaneous microwave ablation of renal cell carcinoma using a high power microwave system: focus upon safety and efficacy. Int J Hyperthermia 2018;34:1077-81.

12. Carberry GA, Smolock AR, Cristescu M, et al. Safety and Efficacy of Percutaneous Microwave Hepatic Ablation Near the Heart. J Vasc Interv Radiol 2017;28:490-7.

13. Onugha OI, Lee JM. Surgical Treatment of Lung Cancer. Cancer Treat Res 2016;170:77-104.

14. Dupuy DE, Zagoria RJ, Akerley W, et al. Percutaneous radiofrequency ablation of malignancies in the lung. AJR Am J Roentgenol 2000;174:57-9.

15. Macchi M, Belfiore MP, Floridi C, et al. Radiofrequency versus microwave ablation for treatment of the lung tumours: LUMIRA (lung microwave radiofrequency) randomized trial. Med Oncol 2017;34:96.

16. Healey TT, March BT, Baird G, et al. Microwave Ablation for Lung Neoplasms: A Retrospective Analysis of Long-
Term Results. J Vasc Interv Radiol 2017;28:206-11.

17. Wolf FJ, Grand DJ, Machan JT, et al. Microwave ablation of lung malignancies: effectiveness, CT findings, and safety in 50 patients. Radiology 2008;247:871-9.

18. Sidoff L, Dupuy DE. Clinical experiences with microwave thermal ablation of lung malignancies. Int J Hyperthermia 2017;33:25-33.

19. Bastianpillai C, Petrides N, Shah T, et al. Harnessing the immunomodulatory effect of thermal and non-thermal ablative therapies for cancer treatment. Tumour biology : the journal of the International Society for Oncodevelopmental Biology and Medicine 2015;36:9137-46.

20. de Baere T, Tselikas L, Catena V, et al. Percutaneous thermal ablation of primary lung cancer. Diagn Interv Imaging 2016;97:1019-24.

21. Nour-Eldin NA, Exner S, Al-Subhi M, et al. Ablation therapy of non-colorectal cancer lung metastases: retrospective analysis of tumour response post-laserinduced interstitial thermotherapy (LITT), radiofrequency ablation (RFA) and microwave ablation (MWA). Int J Hyperthermia 2017;33:820-9.

22. Brace CL, Hinshaw JL, Laeseke PF, et al. Pulmonary thermal ablation: comparison of radiofrequency and microwave devices by using gross pathologic and CT findings in a swine model. Radiology 2009;251:705-11.

23. Carrafiello G, Laganà D, Mangini $M$, et al. Microwave tumors ablation: principles, clinical applications and review of preliminary experiences. Int J Surg 2008;6 Suppl 1:S65-9.

24. Yashiro $H$, Nakatsuka $S$, Inoue $M$, et al. Factors affecting local progression after percutaneous cryoablation of lung tumors. J Vasc Interv Radiol 2013;24:813-21.

25. Ye X, Fan W, Chen JH, et al. Chinese expert consensus workshop report: Guidelines for thermal ablation of primary and metastatic lung tumors. Thorac Cancer 2015;6:112-21.

26. Clasen S, Kettenbach J, Kosan B, et al. Delayed development of pneumothorax after pulmonary radiofrequency ablation. Cardiovasc Intervent Radiol 2009;32:484-90.

27. Zheng A, Wang X, Yang X, et al. Major complications after lung microwave ablation: a single-center experience on 204 sessions. Ann Thorac Surg 2014;98:243-8.

Cite this article as: Chen $\mathrm{B}, \mathrm{Li} \mathrm{W}$, Liu Y, Ren S, Wang L, Wang H, Zhao S, Ye L, He Y, Zhou C. The efficacy and complications of computed tomography guided microwave ablation in lung cancer. Ann Palliat Med 2020;9(5):2760-2765. doi: 10.21037/apm-20-255 